Document downloaded from:

http://hdl.handle.net/10251/141458

This paper must be cited as:

Machado-Olivares, FJ.; PRZEMYSLAW ZAGRAJEK; Ferrando Martín, V.; Monsoriu Serra, JA.; WALTER DANIEL FURLAN (2019). Multiplexing THz Vortex Beams With a Single Diffractive 3-D Printed Lens. IEEE Transactions on Terahertz Science and Technology. 9(1):63-66. https://doi.org/10.1109/TTHZ.2018.2883831

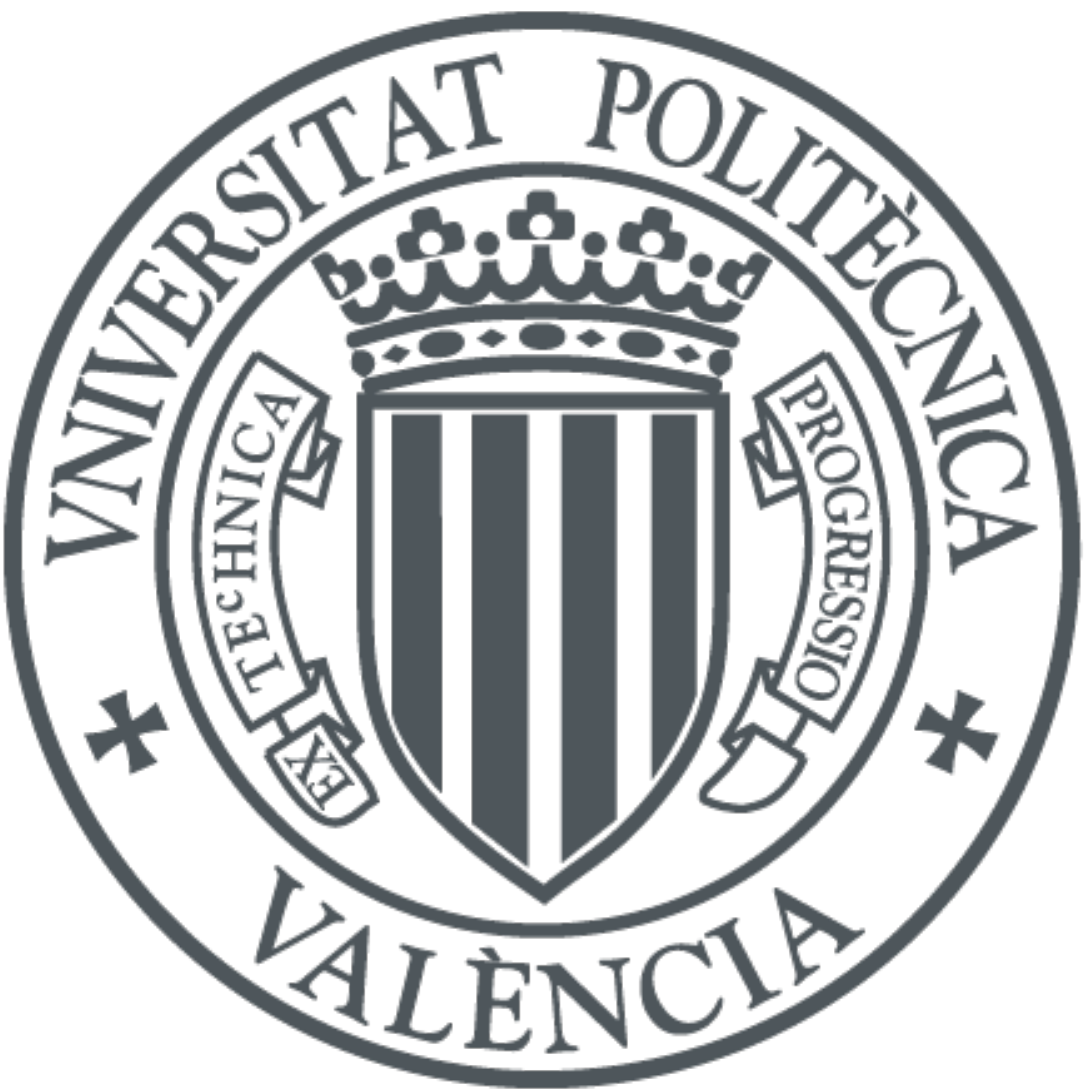

The final publication is available at

https://doi.org/10.1109/TTHZ.2018.2883831

Copyright Institute of Electrical and Electronics Engineers

Additional Information 


\title{
Multiplexing $\mathrm{THz}$ vortex beams with a single diffractive $3 \mathrm{D}$ printed lens
}

\author{
Federico Machado, ${ }^{1}$ Przemysław Zagrajek, ${ }^{2}$ Vicente Ferrando, ${ }^{1}$ Juan A. Monsoriu, ${ }^{1}$ and Walter D. Furlan ${ }^{3 *}$
}

\begin{abstract}
We present a novel method for experimentally generating multiplexed $\mathrm{THz}$ vortex beams by using a single 3D printed element that combines a set of radially distributed spiral phase plates, and a binary focusing Fresnel lens. With this element we have experimentally demonstrated that $\mathrm{THz}$ multiplexing can be tailored to fit within a small space on an optical bench. Results are presented beside numerical simulations, demonstrating the robust nature of the experimental method.
\end{abstract}

Index Terms-Diffraction, diffractive lenses, Vortex lenses, Multiplexed vortex beams

\section{INTRODUCTION}

The singular properties of terahertz radiation, such as good penetration and low scattering through various dielectric materials, non-ionizing photon energy, and broad spectral bandwidth, motivated the growing of $\mathrm{THz}$ photonics. Research in this field was benefited from the development of more efficient emitters, detectors, and optical components such as refractive and diffractive lenses, gratings, beam splitters, polarizers, and retarders [1, 2]. Recently, it has been demonstrated that even low cost 3D printing technology can be employed to construct non-conventional diffractive $\mathrm{THz}$ lenses [3-7]. In this way, technological improvements regarding $\mathrm{THz}$ beam shaping in the form of vortex beams, are of interest because, although such beams have found a large number of applications in the visible domain (e.g., in sensing, microscopy and astronomical imaging, trapping and manipulating of matter, and communication technologies) [8-12], few works have reported applications in the $\mathrm{THz}$ domain.

Vortex beams have orbital angular momentum and propagate with helical phase structure characterized by its azimuthally dependent phase $\operatorname{im} \varphi$, in which $\varphi$ is the transverse azimuthal coordinate and $m$ is known as the topological charge. The fact that $m$ can take any integer value

Manuscript received July 17, 2018; accepted November 12, 2018. Date of publication XXXXX YY, 201Z; date of current version September 7, 2018. This study was supported by the Ministerio de Economía y Competitividad and FEDER (Grant DPI2015-71256-R), Spain, and by the Generalitat Valenciana, (Grant PROMETEO II-2014-072), Spain. Partial support by the National Center for Research and Development in Poland (Grant LIDER/020/319/L-5/13/NCBR/2014) is also acknowledged.

${ }^{1}$ F. Machado, V. Ferrando and J. A. Monsoriu are with Centro de Tecnologías Físicas, Universitat Politècnica de València, Camino de Vera s/n, 46020 Valencia.

${ }^{2} \mathrm{P}$. Zagrajek is with Institute of Optoelectronics, Military University of Technology, Warsaw, Poland.

${ }^{3}$ W. D. Furlan is with Departamento de Óptica y Optometría y Ciencias de la Visión, Universitat de València, E-46100 Burjassot, Spain (e-mail:walter.furlan@uv.es). motivated its use to encode and transmit information [13]. Therefore, due to its high frequency, $\mathrm{THz}$ beams are good candidates for information carrier of the wireless communications. Moreover, vortex beams with different $m$ values can be used as different carriers for multiplexing and transmitting different data streams along the same spatial axis improving the performance of communication systems using electromagnetic waves.

Successful methods used to obtain $\mathrm{THz}$ vortex beams include: off-axis holograms [14], quarter-wave plates coupled to a wire polarizer [15], arrays of wavelength-size V-shaped antennas [16], binary phase axicons with spiral configuration of zones [17, 18, 19], and a photopatterned birefringence liquid crystal [18]. Recently, linearly and circularly polarized vortex Bessel beams were generated by employing a quartz $\mathrm{THz}$ quarter wave plate, a spiral phase plate, and Teflon axicons with different opening angles [21]. A metasurface based method to generate vortex beams using cross shaped resonators was also proposed [22]. However, most of these methods were demonstrated for single vortex beams. Since multiplexing several data channels has been used to significantly increase the data capacity in optical networks [23-25], in this paper, we examine the creation of composite $\mathrm{THz}$ vortex beams representing a superposition of vortices with different topological charges. A multiplexing approach, known as space-division multiplexing [26, 27], has been implemented to a multiplexed vortex THz lens (MVTL) using a $3 \mathrm{D}$ printer. The analysis of the $\mathrm{THz}$ vortices produced by this structure was performed experimentally and numerically for comparison.

\section{DESIGN AND CONSTRUCTION}

Our strategy for generating multiplexed $\mathrm{THz}$ vortex waves using a single element, consists on the combination of two elements represented as the product of two separable functions: one, having a radial phase dependence that can be expressed in terms of a Ronchi-type periodic function with period $p$ in the variable $(r / a)^{2}$ as

$$
\phi(r)=\pi \operatorname{rect}\left[(r / a)^{2}-0.5\right] \operatorname{rect}\left[\frac{\bmod \left[(r / a)^{2}+0.5 p-1, p\right]}{p}\right](1)
$$

where $a$ is the radius of the lens. The other element is a multiplexed spiral zone plate, which only has a phase dependence (linear) on the azimuthal angle. In this element $N$ non-overlapping annular zones are arranged using the space division multiplexing technique. 

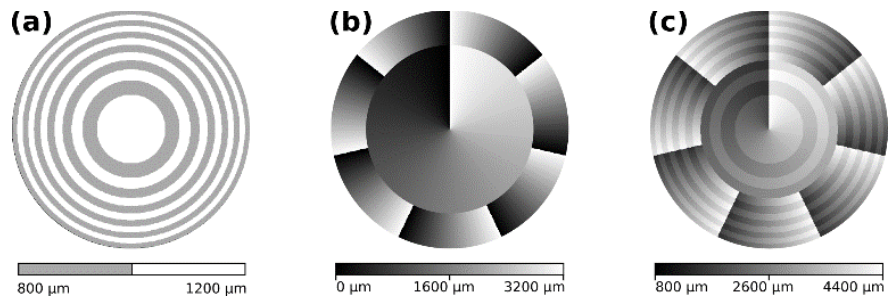

Fig. 1. Design of a multiplexed THz vortex lens. a) Base FZP using (1) with $p=1 / 6, \mathrm{~b}$ ) Multiplexed vortex SPP with topological charges inner part and outer part. c) Composite vortex lens as the superposition of a) and b). Gray levels represent the lens thickness.

$$
\tau(\theta)=\left\{\begin{array}{c}
m_{1} \theta, \quad 0 \leq r<r_{1} \\
\cdots \\
m_{j} \theta, \quad r_{j-1} \leq r<r_{j} \\
\cdots \\
m_{N} \theta, r_{N-1} \leq r<a
\end{array}\right\}
$$

In this way the transmittance of the lens is given by equation $T(r, \theta)=e^{i[\phi(r)+\tau(\theta)]}$.

As a demonstration, we propose a MVTL that consists on the combination of two spiral phase functions with different topological charges $m_{1}$ and $m_{2}$, designed to work at $0.625 \mathrm{THz}$, and made of PA6 polyamide (absorption coefficient $=3.9 \mathrm{~cm}^{-1}$ and refractive index $n=1.6$ ). Other 3D printed material were studied in Ref. [4].

The MVTL composition from its components is shown in Fig. 1. On the one hand, the MVTL (Fig. 1a) was designed with alternate zones of thickness $t_{1}=0.8 \mathrm{~mm}$ and $t_{2}=1.2 \mathrm{~mm}$ which have been calculated to provide a phase shift $\pi$ between the zones for the design wavelength of $\lambda=480 \mu \mathrm{m}$, and using the following expression: $\Delta t=t_{2}-t_{1}=\lambda / 2(n-1)$ On the other hand, the spatially multiplexed $\mathrm{THz}$ Spiral Phase Plate (SPP) (Fig. 1b) has two parts. The inner part, with topological charge $m_{1}=-1$ is a circle of radius $14.67 \mathrm{~mm}$ that covers the 4 inner rings of the FZP. The outer part, with topological charge $m_{2}=7$ has an annular shape with an outer radius $25.4 \mathrm{~mm}$ and covers from the 5 th to the 12th of the FZP. In each part, the thickness of the SPP depends on the azimuthal angle around the center of the SPP, as

$$
h(\theta)=\bmod (m \theta, 2 \pi) \frac{\lambda}{2 \pi(n-1)}
$$

Thus, a total phase shift of $|2 \pi m|$ will be imprinted on the electromagnetic wave by each part of the SPP. Taking into account that this approach requires extreme precision in the pitch of helical surface, in order to reduce the fabrication errors we incremented the thickness in each point of the SPP by a constant factor $k=4$ providing an effective topological charge of $\mathrm{km}$.

In this way for the plate in Fig. $1 \mathrm{~b}), 0 \leq h \leq 3.2 \mathrm{~mm}$. Thus, the final $\mathrm{THz}$ design, shown in Fig. 1c), results as the superposition of the plates in Fig. 1a) and Fig. 1b).

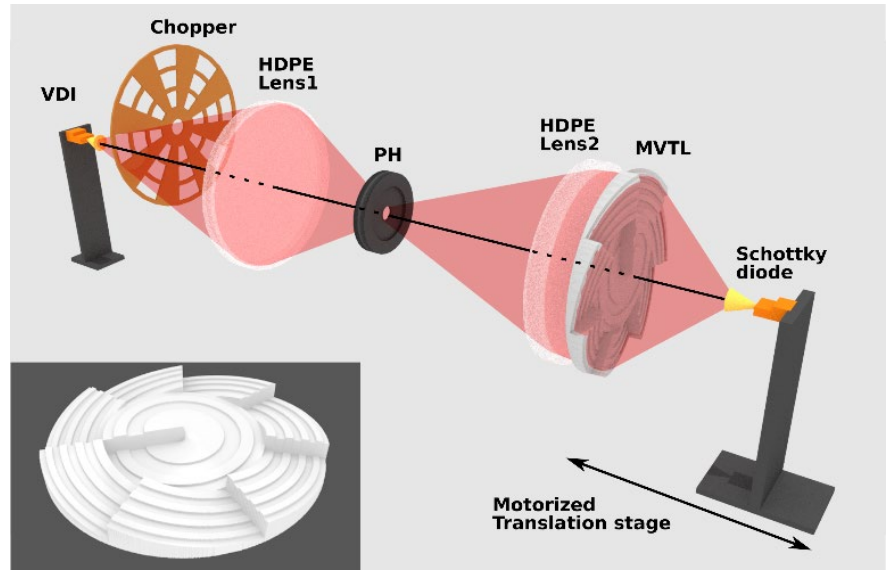

Fig. 2. Experimental setup for obtaining multiplexed $\mathrm{THz}$ vortex beams. The inset shows a CAD design of the MVTL experimental model.

\section{EXPERIMENTAL SETUP}

The experimental setup for obtaining multiplexed $\mathrm{THz}$ vortex beams is shown in Fig. 2. A frequency multiplier based on Schottky diode (Virginia Diodes, Inc. Charlottesville, VA. USA), was used as a source of radiation. The source was equipped with a waveguide, which ends in a horn antenna producing linearly polarized Gaussian beam at $0.625 \mathrm{THz}$. In our case the electric field was perpendicular to the ground. The beam was focused on a pinhole ( $2 \mathrm{~mm}$ diameter) by a high density polyethylene (HDPE) refractive lens. Then, the radiation was collimated, by a second HDPE lens, and directed onto the MVTL. The intensity distribution after the MVTL was scanned with a Schottky diode detector VDI (Virginia Diodes, Inc. Charlottesville, VA. USA) also equipped with horn antenna (WR-1.5), having a $2.4 \mathrm{~mm}$ aperture diameter. As each component of our experimental setup was a passive element that do not changed the polarization of the input beam, the detector was aligned to have the same orientation of the source. The detector was mounted on a 3D motorized stage which allows high precision movement. To measure the signal from the detector, a lock-in system (Stanford Research Systems SR830), based on modulation at $187 \mathrm{~Hz}$ and a mechanical chopper, was used.

The inset in Fig. 2 shows the experimental model, which was designed using a CAD software (blender.org) and constructed with a $0.3 \mathrm{~mm}$ spatial resolution by an online 3D printing service (i.materialise, Leuven, Belgium). The MVTL was made from a polyamide granular powder by selective laser sintering technique. The diameter of the constructed lens was $50.8 \mathrm{~mm}$, and the FZP has the main focal distance (first diffraction order) of $112 \mathrm{~mm}$.

\section{RESULTS}

Beam transverse irradiance profiles provided by the composite helical beam were recorded after the MVTL, along the optical axis in $1 \mathrm{~mm}$ intervals with an accuracy of $2 \mu \mathrm{m}$. Experimental results at five different planes are shown in Fig. 3 in comparison with numerical results computed using the Fresnel-Kirchhoff nonparaxial scalar diffraction theory [6]. 


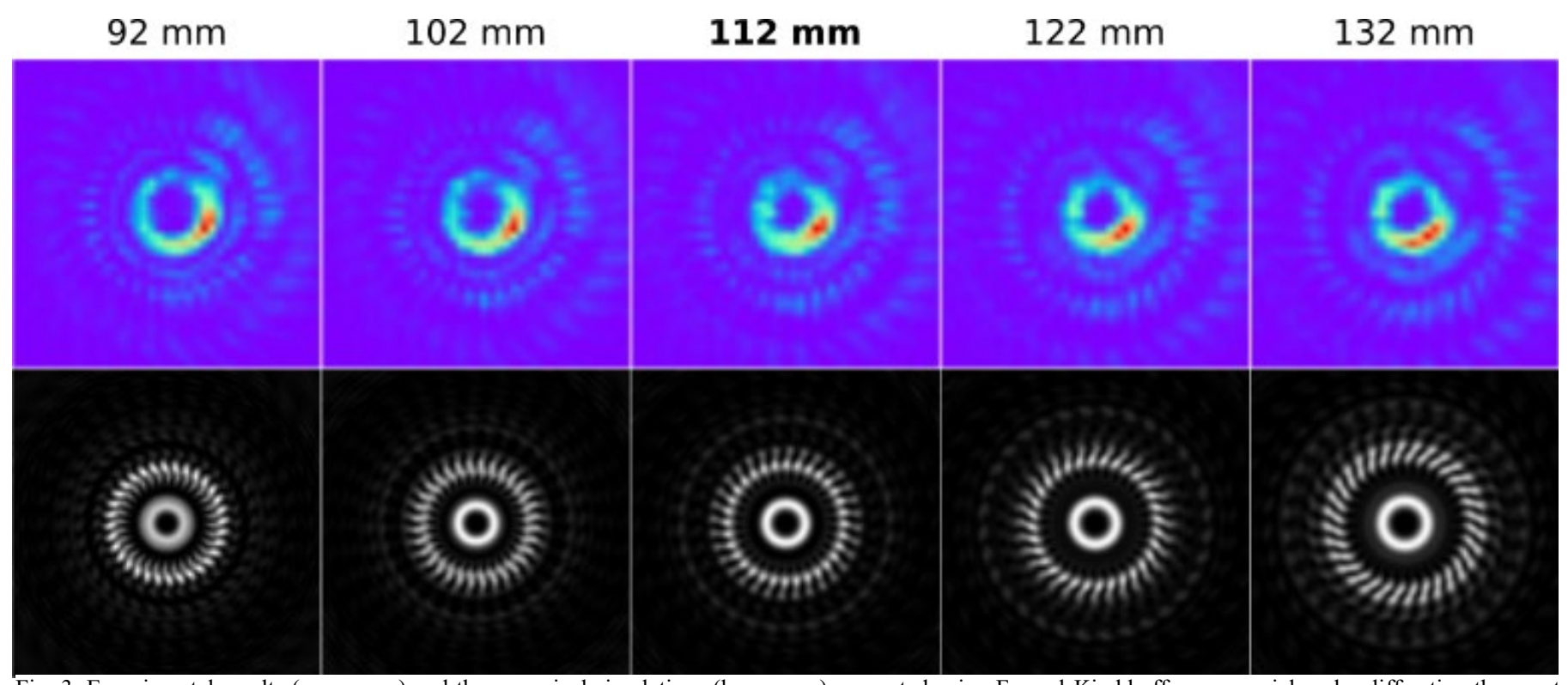

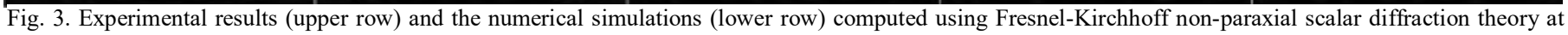
different transverse planes around the MVTL focal plane $(z=112 \mathrm{~mm})$.

As expected the two main vortices are in focus at the MVTL focal distance, the inner vortex corresponds to the topological charge $m_{1}=-1$, while the outer vortex corresponds to the topological charge $m_{2}=7$. The last one also exhibits a complex structure with a cosenoidal azimuthal variation. In fact, $k\left(m_{2}-m_{1}\right)=32$ lobes appear, as it was expected by the theoretical analysis [28].

Despite of the differences between the physical lens and the theoretical design, due to the limitations of the $3 \mathrm{D}$ printer resolution (especially near the center of the plate), the agreement between theory and experiment can be appreciated in Fig.3. Additional vortices, seen in the experimental results, are the consequence of non-integer steps heights of the lens designed for only one spectral component. Other sources of discrepancy between experimental and theoretical results are the inhomogeneities of the index of refraction and the finite size of the lens. Multiple reflections and standing waves in the MVTL, may also lead to azimuthal modulations in intensity [29].

\section{CONCLUSIONS}

We have demonstrated the feasibility of realizing $\mathrm{THz}$ multiplexed vortex beams, with 3D printing technology. PA6 phase lenses were constructed and tested using $0.625 \mathrm{THz}$ beam in a simple experimental $\mathrm{THz}$ setup. The experimental results were in good agreement with the simulations despite some sources of errors, demonstrating the robustness of our proposal. In this way, this work extends to the $\mathrm{THz}$ domain previous use of phase optical elements in the visible domain [30] that could find many applications in $\mathrm{THz}$ technologies. For instance, there have been recent growing interest in applying vortex beams to wireless communications [31] and through mode multiplexing, vortex beams can tremendously increase the capacity of communication systems.

These novel techniques, developed for utilizing $\mathrm{THz}$ spectrum can be improved to achieve very high efficiency. In this sense, it has been demonstrated that dependencies of the vortex radius and width on topological charge can be controlled [30], and, therefore, multiplexed $\mathrm{THz}$ vortex rings can be produced efficiently.

\section{REFERENCES}

[1] D. Mittleman, Sensing with Terahertz Radiation. Berlin, Germany, Springer, 2003.

[2] M. Tonouchi, "Cutting-edge terahertz technology," Nat. Photonics, vol. 1, pp. 97-105, Feb. 2007.

[3] X. Wei, C. Liu, L. Niu, Z. Zhang, K. Wang, Z. Yang, and J. Liu, "Generation of arbitrary order Bessel beams via 3D printed axicons at the terahertz frequency range," Appl. Opt., vol. 54, pp. 10641-10649, Dec. 2015.

[4] W. D. Furlan, V. Ferrando, J. A. Monsoriu, P. Zagrajek, E. Czerwinska, and M. Szustakowski, "3D printed diffractive terahertz lenses," Opt. Lett., vol. 41, no. 8, pp. 1748-1751, Apr. 2016.

[5] C. Liu, L. Niu, K. Wang, and J. Liu, "3D-printed diffractive elements induced accelerating terahertz Airy beam," Opt. Express, vol. 24, no. 25, pp. 29342-29348, Dec. 2016.

[6] W. D. Furlan, F. Machado, J. A. Monsoriu, and P. Zagrajek, "Terahertz Sieves," IEEE T. THz Sci. Techn., vol. 8, no. 1, pp. 140-143. Jan. 2018.

[7] B. Mirzaei et al., "Efficiency of multi-beam Fourier phase gratings at $1.4 \mathrm{THz}$," Opt. Express, vol. 25, no. 6, pp. 6581-6588, Mar. 2017.

[8] F. S. Roux, "Distribution of angular momentum and vortex morphology in optical beams," Opt. Commun., vol. 242 no. 1-3, pp. 45-55, Nov. 2004. 
[9] G. Gbur, and T. D. Visser, "Phase singularities and coherence vortices in linear optical systems," Opt. Commun., vol. 259, no. 2, pp. 428-435, Mar. 2006.

[10] W. M. Lee, X. C. Yuan, and W. C. Cheong, "Optical vortex beam shaping by use of highly efficient irregular spiral phase plates for optical micromanipulation," Opt. Lett., vol. 29, no. 15, pp. 1796-1798, Aug. 2004.

[11] S. H. Tao, X.-C. Yuan, J. Lin, and R. Burge, "Sequence of focused optical vortices generated by a spiral fractal zone plates," Appl. Phys. Lett., vol. 89, no. 3, pp. 031105, Jul. 2006.

[12] W. D. Furlan, F. Giménez, A. Calatayud, and J. A. Monsoriu, "Devil's vortex-lenses," Opt. Express, vol. 17, no. 24, pp. 21891-21896, Nov. 2009.

[13] J. Wang, "Advances in communications using optical vortices," Photon. Res., vol. 4, no. 5, pp. B14-B28, Oct. 2016.

[14] Z. Xie, X. Wang, J. Ye, S. Feng, W. Sun, T. Akalin, andY. Zhang, "Spatial terahertz modulator," Sci. Rep., vol. 3, pp. 3347, Nov. 2013.

[15] R. Imai, N. Kanda, T. Higuchi, K. Konishi, and M. Kuwata-Gonokami," Generation of broadband terahertz vortex beams," Opt. Lett., vol. 39, no. 13, pp. 3714-3717, Jul. 2014.

[16] J. He, X. Wang, D. Hu, J. Ye, S. Feng, Q. Kan, and Y. Zhang, "Generation and evolution of the terahertz vortex beam," Opt. Express, vol. 21, no. 17, pp. 20230-20239, Aug. 2013.

[17] K. Miyamoto, K. Suizu, T. Akiba, and T. Omatsu, "Direct observation of the topological charge of a terahertz vortex beam generated by a Tsurupica spiral phase plate," Appl. Phys. Lett., vol. 104, no. 26, pp. 261104, Jul. 2014.

[18] B. A. Knyazev, Y. Y. Choporova, M. S. Mitkov, V. S. Pavelyev, and B. O. Volodkin, "Generation of Terahertz Surface Plasmon Polaritons Using Nondiffractive Bessel Beams with Orbital Angular Momentum," Phys. Rev. Lett., vol. 115, no. 16, pp. 163901, 2015.

[19] Y. Y. Choporova, B. A. Knyazev, G. N. Kulipanov, V. S. Pavelyev, M. A. Scheglov, N. A. Vinokurov, B. O.Volodkin, and V. N. Zhabin, "High-power Bessel beams with orbital angular momentum in the terahertz range," Phys. Rev. A, vol. 96, no. 2, pp. 023846, 2017.

[20] S. Ge, P. Chen, Z. Shen, W. Sun, X. Wang, W. Hu, Y. Zhang, and $\mathrm{Y} . \mathrm{Lu}$, "Terahertz vortex beam generator based on a photopatterned large birefringence liquid crystal," Opt. Express, vol. 25, no. 11, pp. 12349-12356, May. 2017.
[21] Z. Wu, X. Wang, W. Sun, S. Feng, P. Han, J. Ye, Y. Yu, Y. Zhang, "Vectorial diffraction properties of THz vortex Bessel beams," Opt. Express, vol. 26, pp. 1506, 2018.

[22] R. Dharmavarapu, S. Hock Ng, S. Bhattacharya, S. Juodkazis, "All-dielectric metasurface for wavefront control at terahertz frequencies," Proc. SPIE, vol. 10456, Nanophotonics Australasia 2017, 104561W, Jan. 2018.

[23] S. Yu, "Potentials and challenges of using orbital angular momentum communications in optical interconnects," Opt. Express, vol. 23, no. 3, pp. 3075-3087, Feb. 2015.

[24] T. Lei, M. Zhang, Y. R. Li, P. Jia, G. N. Liu, X. G. Xu, Z. H. Li, C. J. Min, J. Lin, C. Y. Yu, H. B. Niu, and X. C. Yuan, "Massive individual orbital angular momentum channels for multiplexing enabled by Dammann gratings," Light-Sci. Appl., vol. 4, pp. e257, Mar. 2015.

[25] J. Wang, J. Y. Yang, I. M. Fazal, N. Ahmed, Y. Yan, H. Huang, Y. Ren, Y. Yue, S. Dolinar, M. Tur, and A. E. Willner, "Terabit freespace data transmission employing orbital angular momentum multiplexing," Nat. Photonics, vol. 6, no. 488-496, Jun. 2012.

[26] G. Li, N. Bai, N. Zhao, and C. Xia, "Space-division multiplexing: the next frontier in optical communication," Adv. Opt. Photon., vol. 6, no. 4, pp. 413-487, Dec. 2014.

[27] X. Wei, L. Zhu, Z. Zhang, K. Wang, J. Liu and J. Wang, "Orbit angular momentum multiplexing in 0.1-THz freespace communication via 3D printed spiral phase plates," Proc. Conference on Lasers and Electro-Optics (CLEO) Laser Science to Photonic Applications 2014, San Jose (CA), pp. 1-2, 2014.

[28] Z. Bouchal, V. Kollarova, P. Zemanek, and T. Cizmar, "Orbital angular momentum of mixed vortex beams," Proc. SPIE, no. 660907, pp. 1-8, Apr. 2007.

[29] M. V. Berry, "Optical vortices evolving from helicoidal integer and fractional phase steps," J. Opt. A: Pure Appl. Opt., vol. 6, no. 2, pp. 259-268, Jan. 2004.

[30] C.-S. Guo, X. Liu, J.-L. He, and H.-T. Wang, "Optimal annulus structures of optical vortices," Opt. Express, vol. 12, no. 19, pp. 4625-4634, Sep. 2004.

[31] E. Willner, H. Huang, Y. Yan, Y. Ren, N. Ahmed, G. Xie, C. Bao, L. Li, Y. Cao, Z. Zhao, J. Wang, M. P. J. Lavery, M. Tur, S. Ramachandran, A. F. Molisch, N. Ashrafi, and S. Ashrafi, "Optical communications using orbital angular momentum beams," Adv. Opt. Photon., vol. 7, no. 1, pp. 66-106, Mar. 2015. 


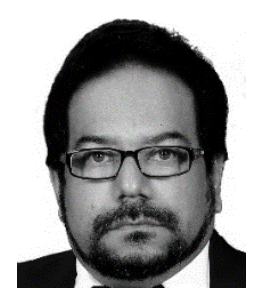

Federico Machado was born in El Salvador in 1970. He received the B.S. degree in electrical engineering from Universidad Politécnica de El Salvador in 1993 and M.S. degree on management of renewable resources from Universidad Don Bosco (El Salvador) in 2013. He is currently pursuing the Ph.D. degree at Universidad Politécnica de Valencia. He worked at Universidad Don Bosco for 15 years in different positions, teaching subjects related to electrical, electronics and renewable resources. His experience is focused on energy efficiency and designing electronic systems to support educational systems.

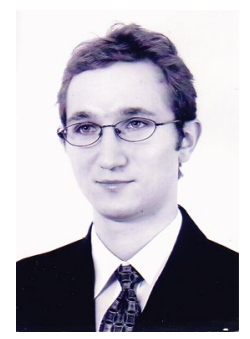

Przemyslaw Zagrajek was born in 1979. He received his Master degree in Physics, at The Faculty of Applied Physics and Mathematics in Warsaw University of Technology in 2004 and Ph.D. degree in Institute of Physics, Polish Academy of Sciences. Warsaw, Poland in 2012. From 2012 he is an Assistant Professor with the Laboratory of Terahertz Technology at Institute of Optoelectronics at Military University of Technology, Warsaw, Poland. His areas of interest are spintronics and physics of nanodevices.

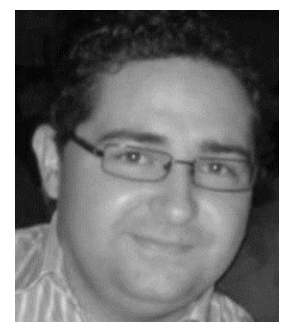

Vicente Ferrando was born in Valencia, Spain, in 1986. He received the B.S. degree en Physics and the M.S. degree in advanced physics (Photonics) from Universitat de València (UV), Spain, in 2010 and 2012, respectively. $\mathrm{He}$ achived his $\mathrm{PhD}$ degree at Universitat Politècnica de València (UPV) in 2017. Since 2011, he is working as a researcher at the Diffraction Optics Group (DiOG) (UV and UPV). His current main research is to study the optical properties of aperiodic diffractive lenses and their applications.

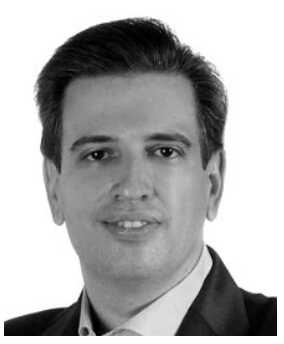

Juan A. Monsoriu was born in Valencia, Spain, in 1975. He received the B.S. degree in physics, M.S. degree in Optics, and Ph.D. degree in Physics from the Universidad de Valencia (UV), Spain, in 1998, 2000, and 2003, respectively. In 2000 he joined the Universidad Politécnica de Valencia (UPV), Spain, where he is currently Full Professor of Applied Physics. His research was performed at UV, UPV, Universidad de Málaga (Spain), University of Bath (UK), and Universidad de Buenos Aires (Argentina). His main research interests include numerical simulations for the design of micro structured optoelectronic systems and aperiodic optical devices.

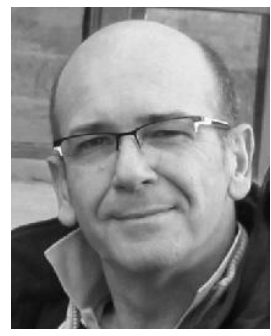

Walter D. Furlan received his MS and $\mathrm{Ph} . \mathrm{D}$. degrees in physics from the University of La Plata, Argentina, in 1984 and 1988, respectively. Until 1990 he worked at the Centro de Investigaciones Opticas (CIOp), Argentina. At the end of that year he joined the Optics Department of the University of Valencia, Spain, where he is currently professor of Optics. His research has been developed in the field of optics in two well differentiated areas. On the one hand, he investigated on theory and applications of phase-space representations (Wigner Distribution Function, Ambiguity Function, etc.). In these topics he published more of 25 papers in refereed journals and a chapter in the book Phase-Space Optics: Fundamentals and Applications (McGraw Hill Professional, 2009). On the other hand, mainly in the last ten years, he focused on the study of the properties of nonconventional diffractive optical elements. He co-authored more than 30 papers and 3 patents related with these topics. Currently he is Full Professor at the University of Valencia, and co-director of the Diffractive Optics Group (DiOG). Dr. Furlan is a member of the European Optical Society. 